\title{
NASA'S TECHNOLOGY TRANSFER PROGRAM FOR THE EARLY DETECTION OF BREAST CANCER
}

\author{
Gregory Schmidt, Mary Anne Frey, Ph.D., Joan Vernikos, Ph.D. NASA Headquarters, Washington DC 20546. \\ Daniel Winfield, Research Triangle Institute, Research Triangle Park, North Carolina 27709.
}

\begin{abstract}
The National Aeronautics and Space Administration (NASA) has led the development of advanced imaging sensors and image processing technologies for space science and Earth science missions. NASA considers the transfer and commercialization of such technologies a fundamental mission of the agency. Over the last two years, efforts have been focused on the application of aerospace imaging and computing to the field of diagnostic imaging, specifically to breast cancer imaging. These technology transfer efforts offer significant promise in helping in the national public health priority of the early detection of breast cancer.

KEY WORDS:

digital mammography, breast cancer, $\mathrm{X}$-ray $\mathrm{CCD}$
\end{abstract}

\section{INTRODUCTION}

Breast cancer is the leading cause of death among women between the ages of 35 and 50 . Over 182,000 new cases of this disease were diagnosed in the U.S. in 1993 with 46,000 deaths, and statistics show that one in eight women will develop breast cancer at some time in their lives. Health care costs associated with breast cancer in the U.S. total more than $\$ 8$ billion per year [1]. The early detection of this disease through the application of advanced imaging technologies has been identified by the National Cancer Institute (NCI) as a high priority [2].

NASA's Astronomy and Earth Sciences missions have driven the development of many imaging technologies. These missions and breast imaging have many common requirements: fine resolution to capture great detail in images, a wide dynamic range to capture structures spanning orders of magnitude in brightness, and sensitivity to low light levels to reduce exposure requirements. Imaging breast tissue faces many technical challenges, from resolving finely detailed structures such as microcalcifications (an early indicator of breast cancer) to being able to provide images simultaneously showing fibrous tissue and fatty tissue, which have a great deal of difference in their X-ray transmission levels. In addition, the difficulties in diagnosing disease from mammograms result in a large variability between individual physicians, which could be aided by intelligent systems.

One NASA-driven development has already found its way into clinical use as part of the LORAD stereotactic needle biopsy system. The charge-coupled device (CCD) camera used in this system was originally designed and built for use in the Hubble Space Telescope Imaging Spectrograph, and provides a high-resolution, high-contrast image in real time to guide a physician in the accurate collection of a biopsy sample from suspicious imaged breast lesions. The Hubble $C C D$, coupled with a high-speed phosphor screen, gives greatly increased sensitivity, contrast and resolution over previous methods. The result is a less traumatic, lower cost ( $\$ 800$ vs. $\$ 2,500$ typically for surgical biopsy), non-surgical biopsy procedure for the more than 500,000 American women who undergo breast biopsies each year.

\section{NCI-NASA PARTNERSHIP}

In 1993, NASA and the National Cancer Institute (NCI) signed an Agreement to work together to identify applications of emerging aerospace technologies to solve important needs in diagnostic imaging. The initial focus of this partnership has been on the national priority of early breast cancer detection.

The NASA Technology Applications Team at Research Triangle Institute assisted NASA and NCI in searching for applicable technologies from throughout NASA and other Federal laboratories. Forty-three technologies from the aerospace and defense community were evaluated for their potential contribution to digital image acquisition, computeraided diagnosis, and telemammography.

Workshops in May 1993 and May 1995 have served as a forum for Federal laboratory and contractor scientists and engineers to establish a dialogue with professionals from the medical imaging industry and academic community [3]. As a result of the initial workshop, a number of R\&D partnerships have been formed, and NASA and NCI joined to issue a Program Announcement in requesting proposals for technology transfer to digital mammography.

\section{X-RAY DETECTOR DEVELOPMENT}

Following a competitive, peer-review process, NASA and NCI have supported five projects that offer the promise of developing direct digital mammography systems with high resolution and wide field of view. Direct digital methods offer the advantages of greater sensitivity to $\mathrm{X}$-rays, a wider dynamic range allowing imaging of both dense and fatty breast tissues, and improved image quality as a result of the higher contrast provided by the image detector when compared to $x$-ray film.

First, NASA and NCI are supporting a CCD/Scintillation Fiber Detector project that will use specially-doped plastic scintillation fibers to convert $x$-rays transmitted through the breast into blue light. This light is then imaged by customdesigned charge coupled devices designed for ultrahigh sensitivity to blue light. The project marries the scintillation fiber expertise of Nanoptics, Inc. with CCD imaging experience of the NASA Jet Propulsion Laboratory, developed to support planetary exploration missions, and will be tested at the University of South Florida $[4,5]$.

The second project involves the testing and prototype development of silicon pixel detectors developed by Nova R\&D, Inc. and Hughes Aerospace. Development of silicon 
pixel detectors was funded by NASA, the U.S. Army, and the Ballistic Missile Defense Organization for both spacebome sensors and non-destructive radiography of munitions. These detectors have the advantage of being directly sensitive to $x$-rays (no scintillation material is required) and are cheaper to produce than CCDs since they are fabricated using CMOS technology. Tests at the University of Toronto will determine their suitability for use in a digital mammography system [6]. In each of these first two projects, prototype digital mammography units will be fabricated and tested over the next 1 to 2 years with each achieving 50 micron or better pixel resolution over a $24 \times 30 \mathrm{~cm}$ image area.

In the third project, the NCI is supporting work being conducted at NASA's Langley Research Center and the University of Virginia to develop CCD Mosaic detectors [7]. This project is developing an area mosaic of charge-coupled device (CCD) arrays to achieve a large field of view. By taking 3 images from slightly different positions, a large format, mosaic image is generated with high resolution. The CCD mosaic device will result in a 18 by $24 \mathrm{~cm}$ image size with 16-18 line-pairs per millimeter resolution, corresponding to a 30 micron pixel size. This detector offers excellent image quality and short image acquisition times and has the ability to be retrofitted to existing hospital mammography units. This technology was developed as a follow-on to Langley's remote atmospheric sensing program, and a prototype system demonstration is underway.

Fourth, the University of Massachusetts has teamed with Loral Fairchild Imaging Sensors and NASA Goddard Space Flight Center to develop a "digital mammographic cassette." The primary objective is to develop a high performance imaging module offering high quality digital imaging capability to the average mammographic facility without major modification of existing equipment. While not providing full-field mammography, the imaging module will be designed to provide diagnostic quality images both for localization and spot views.

The final project seeks to transfer over 10 years of R\&D into high resolution CCDs for macromolecular crystallography to digital mammography, resulting in a modular, scaleable, high performance system for full breast imaging. Researchers from the University of Virginia and Brandeis University are developing a detector with high quantum efficiency, large dynamic range, and near real-time readout. Similar to the mosaic technique being pursued by NASA Langley, these CCD detectors will be configured into an area array to provide full breast coverage.

\section{APPLICATIONS OF SETI}

NASA's SETI (Search for Extraterrestrial Intelligence) Program was faced with the challenge to develop high performance methods for detecting weak radio signals buried in cosmic noise, a problem analogous to the early detection of breast cancer. Methods developed in response to this challenge were 10 times more sensitive and processed data 100 times faster than any detection algorithms available at the time of their development. Generalizations of these methods may generate superior methods for finding early signs of breast cancer in mammograms digitized from $x$-ray film and those produced directly using CCDs. NASA is funding a one year study of this hypothesis using a large database of mammogram images compiled and analyzed by the Moffin Cancer Center at the University of South Florida. Early results from this study indicate that statistical analysis through wavelet transforms can be used to define healthy tissue and then automatically recognize diseased tissue, thus increasing the efficiency of breast cancer screening. The study is currently focused on comparing this work with traditional computerized and human-assisted cancer detection methods.

\section{SUMMARY}

NASA is focusing efforts on transferring relevant aerospace technologies to create improved quality $x$-ray images that will enable detection of smaller and more subtle features found in the early stages of breast cancer. Through its parmerships with the $\mathrm{NCl}$, other government agencies, universities and physicians concemed with imaging technologies and women's bealth, significant progress is being made in the efforts toward the early detection of breast cancer.

\section{ACKNOWLEDGMENTS}

The authors wish to acknowledge Faina Shtern, M.D., Special Assistant to the Director of $\mathrm{NCI}$, and Susan Blumenthal, M.D., Deputy Assistant Secretary for Health, Women's Health, for their support of and interest in NASA's technology transfer efforts. Kent Cullers, Ph.D. of the SETI Institute is also gratefully acknowledged for his visionary ideas and tireless pursuit in applying his advanced signal processing methods to women's health.

\section{REFERENCES}

1. American Cancer Society, Cancer Facts and Figures. Atlanta, GA. 1993.

2. Shtern, F. Digital mammography and related technologies: a perspective from the National Cancer Institute. Radiology 183:629-630, 1992.

3. Winfield, D.L., et al. Technology transfer in digital mammography, Investigative Radiology 29:507-515, April 1994.

4. Jing, Z., Huda, W., Walker, J.K., Choi, W.Y. Image characteristics of plastic scintillating fiber screens for mammography, SPIE Proceedings Vol. 2708, Physics of Medical Imaging, Feb. 1996.

5. Hasan, S.Y. The Design of a Real Time Flat-Fielding Unit for a TDI Mode CCD Mammography Camera, Degree of Engineer Thesis, Dept. of Industrial Engineering, Univ. of Florida, May 1996.

6. Henry, J.M., Yaffe, M.J., Pi, B., Venzon, J.E., Augustine, F., Tumer, T.O. Solid state x-ray detectors fú digital mammography. Proceedings of the Society of PhotoOptical Instrumentation Engineers 2432, 392-401, 1995.

7. Jalink, A., McAdoo, J., Halama, G., Liu, H. CCD

Mosaic technique for large field digital mammography, IEEE Transactions in Medical Imaging, Volume 15, No. 4, pp. 260-267. August 1996. 